\title{
Relación entre la flexión dorsal de tobillo y la flexibilidad de la columna medial
}

\author{
Relationship between dorsal ankle flexion and medial column flexibility \\ Noelia Sales Picher ${ }^{1}$ y Enrique Sanchis Sales ${ }^{2}$ \\ ${ }^{1}$ Graduada en Podología. Universitat de València. Valencia, España. ${ }^{2}$ Departamento de Enfermería y Podología. Universitat de València. \\ Valencia, España
}

\section{Palabras clave:}

Postura del pie, columna medial, marcha, flexión dorsal de tobillo, test de Lunge, navicular drop, navicular drift.

\section{Resumen}

Objetivos: Debido a su importancia durante las actividades de carga, el estudio de la articulación de tobillo recibe gran atención en la literatura. Es muy frecuente analizar el rango de flexión dorsal de tobillo, ya que se piensa que una incorrecta movilidad puede afectar a la función de la extremidad inferior y del pie, más concretamente sobre el arco longitudinal interno.

Pacientes y métodos: Para la valoración de la flexión dorsal de tobillo se utilizó el test de Lunge. Para valorar la movilidad de la columna medial se calculó el desplazamiento y el descenso del navicular mediante las pruebas de navicular drop y navicular drift. Para analizar la postura del pie se utilizó el índice de la postura del pie.

Resultados: Se analizaron un total de 57 adultos sanos. Se encontró una relación lineal e inversa entre la flexión de tobillo y la flexibilidad de la columna medial. Respecto al índice de postura del pie no se observó ninguna relación con el test de Lunge, pero sí que influye en la flexibilidad de la columna medial.

Conclusión: El presente estudio ha encontrado correlación entre el rango de flexión dorsal de tobillo y la estabilidad de la columna medial medida clínicamente, y entre la posición del pie y la estabilidad de la columna medial en sujetos sanos. Se ha encontrado que a mayor flexión dorsal de tobillo, parece existir menor flexibilidad de la columna medial.

\section{Key words:}

Foot posture, medial column, gait, dorsiflexion ankle, Lunge's test, navicular drop, navicular drift.

\section{Abstract}

Objectives: Due to its importance during weightbearing activities the study of the ankle joint has recieved much importance in literature. It is very frequent to analyze the range of dorsiflexion of the ankle because it is thought that an incorrect range of motion can affect the function of the lower limb and the foot, to be more concrete, internal longitudinal arch.

Patients and method: To value the dorsiflexion of the ankle we used the Lunge's test. To assess the mobility of the medial column, the navicular was measured using the drop and drift navicular tecniques. Foot posture was studied using the Foot Posture Index.

Results: A total of 57 healthy individuals were included in the study. It's was found a lineal and inverse relation between the ankle flexion and the flexibility of the medial column. However, no relationship was found between the Foot Posture Index and the Lunge test, but relationship was found with the navicular drop and drift.

Conclusion: The present study has found a correlation between the range of ankle dorsiflexion and medial column stability measured clinically, and between foot position and medial column stability in healthy subjects. Finding that the greater the ankle dorsiflexion, there appears to be less flexibility of the medial column. 


\section{INTRODUCCIÓN}

En la actualidad el estudio de la articulación tibioperoneoastragalina (ATPA) o articulación de tobillo junto con la columna interna del pie sigue siendo de gran interés para el análisis biomecánico de la marcha humana. El tobillo es una de las estructuras más complejas e importantes del cuerpo humano ${ }^{1-3}$. La ATPA está formada por la unión del astrágalo con la tibia y el peroné ${ }^{1,4,5}$. Esta articulación se caracteriza por poseer movimientos en los tres planos del espacio. El plano que presenta más rango de movimiento es el plano sagital, con $20-30^{\circ}$ de flexión dorsal (FD) con la rodilla extendida y de $30-50^{\circ}$ de flexión plantar (FP) ${ }^{1}$. La ATPA tiene mucha influencia en el patrón de la marcha y parece ser que está bastante relacionada con la aparición de determinadas patologías como la fascitis plantar, hallux limitus o rigidus, hallux valgus, metatarsalgias, tendinopatías del tendón de Aquiles, esguinces de tobillo y síndrome de estrés tibial, entre otras ${ }^{3,5-8}$. Actualmente, la evaluación de la FD y FP de la ATPA se realiza de forma rutinaria en las exploraciones biomecánicas de los pacientes. Es muy frecuente evaluar el rango de movilidad del tobillo en descarga, y es poco frecuente hacerlo en carga ${ }^{3,4-6,9,10}$. Numerosos estudios afirman que es más fiable realizar una medición de la ATPA en carga, ya que es la mejor forma de valorar cómo se comporta dicha articulación durante la dinámica ${ }^{3,9,10}$. El método o prueba más utilizado en la literatura y que muestra un alto coeficiente de fiabilidad para la evaluación de la FD de la ATPA en carga es el test de Lunge. En la literatura ha sido utilizado para analizar si existe relación entre la limitación de la FD y la etiología de otras patologías que afecten a pie y tobillo $3,9,10$.

Otro elemento importante para estudiar en la biomecánica y en la marcha humana es el estudio de la flexibilidad de la columna medial, cuyo elemento más importante es el arco longitudinal interno (ALI $)^{11}$. La columna medial está formada por el astrágalo, el escafoides, las tres cuñas y el primer, segundo y tercer metatarsianos ${ }^{1,11}$. Durante la marcha, la columna medial y en especial el ALI deben poseer la capacidad adecuada de deformarse y recuperarse cuando actúen las fuerzas reactivas del suelo sobre el pie ${ }^{11}$. Por ello es necesario valorar la flexibilidad de la columna medial para conocer su correcto funcionamiento durante la marcha. En la literatura se han descrito diferentes pruebas para la valoración del ALI, siendo las pruebas del navicular drop (NDrop), navicular drift (NDrift) e índice de arco las que presentan un mayor índice de fiabilidad para la valoración del ALI ${ }^{9,12-17}$. Existen factores que parecen influir en la flexibilidad de la columna medial, como podrían ser la FD del tobillo, las fuerzas reactivas del suelo durante las actividades de carga del pie y la postura del pie, entre otros. Para valorar la postura del pie se han descrito múltiples pruebas, y la más utilizada en la actualidad es el índice de la postura del pie (IPP) $)^{1,3,18-23}$. Este estudio tiene como objetivo analizar la relación que existe entre la FD de tobillo, la flexibilidad de la columna medial y la postura del pie.
Nuestro estudio pretende centrarse en la valoración de la FD de la ATPA junto con la flexibilidad de la columna medial y la postura del pie. Trabajos como los de Alfaro Santafé y cols. $2017^{3}$, Williams y cols. $2014^{4}$, Hall y cols. $2017^{6}$, Jung y cols. $2009^{7}$ y Langarika-Rocafort y cols. $2017^{9}$, entre otros, dan suma importancia a ambos elementos en el estudio de la marcha humana. Además, una alteración de estos factores puede verse relacionada con la aparición de determinadas patologías del pie y con un patrón alterado de la marcha.

\section{PACIENTES Y MÉTODOS}

\section{Población de estudio}

Se realizó un estudio observacional, descriptivo, de corte transversal y prospectivo, de 57 sujetos sanos de entre 20 y 50 años, entre febrero y marzo de 2017. Los sujetos del estudio eran alumnos que cursaban académicamente tercer y cuarto curso de grado en podología del curso 2017-2018 en la Universitat de València. Se incluyeron solo los sujetos sanos, sin afectaciones neuromusculares, sin lesiones en el pie o la pierna que tuvieran dolor o les impidiesen la normal movilización, sin antecedentes quirúrgicos en el pie, y con un IPP normal, pronado y supinado. Previamente a la participación en el estudio todos los sujetos firmaron un consentimiento informado, y la investigación fue aprobada por el Comité de Bioética (número procedimiento: H1512121644041) de la Universitat de València (Valencia, España).

\section{Medición de variables}

Para la obtención de los datos, se sometió a los sujetos a una serie de pruebas biomecánicas. Primero se obtuvieron datos antropométricos de cada participante, como la edad, sexo, peso y talla. Todos los sujetos permanecieron en bipedestación en posición relajada del calcáneo en apoyo. En primer lugar, se realizó el IPP tal y como describieron Redmond y cols. en 2006', para determinar la postura en ambos pies. A continuación se calcularon los grados de FD de tobillo en carga de ambos pies mediante el test de Lunge descrito por Bennell y cols. ${ }^{10}$ en 1998. Se midieron los grados de FD utilizando una regla goniómetra de plástico con semicírculo graduado, tal y como se muestra en la Figura 1. Se cuantificaron los grados de inclinación de la tibia con el inclinómentro de la aplicación brújula del sistema operativo IOS de Apple que se encuentra en el Iphone. Posteriormente se realizó a todos los sujetos el NDrop descrito por Brody y cols. en 1982 y NDrift, tal y como explica Menz ${ }^{16}$ en 1998, para medir el desplazamiento y descenso del navicular con papel milimetrado y cuantificar la diferencia en centímetros entre la posición relajada del calcáneo en apoyo y la posición neutra, tal y como se describe en la Figura 2. La obtención de los datos del estudio la realizó un explorador, alumno de cuarto curso del grado de podología, que había sido instruido previamente para la realización de las mediciones. El valor del estudio se obtuvo a partir de una única medición. 


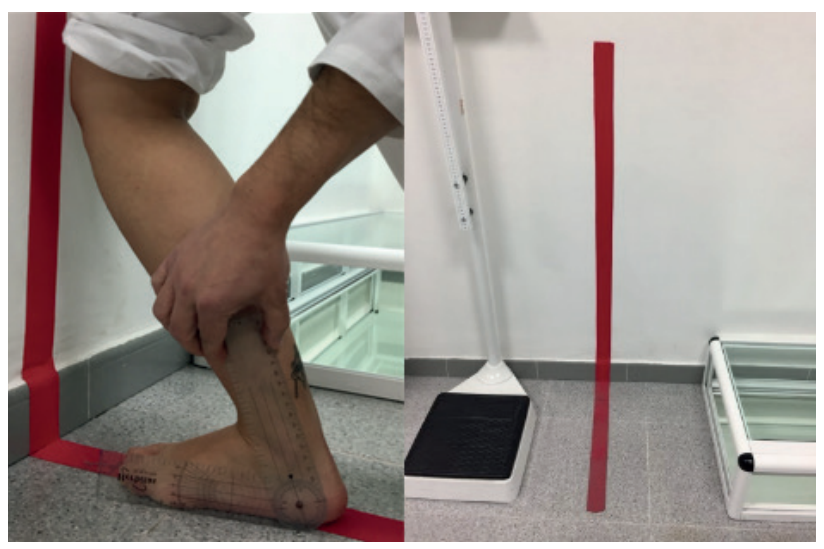

Figura 1. Test de Lunge.
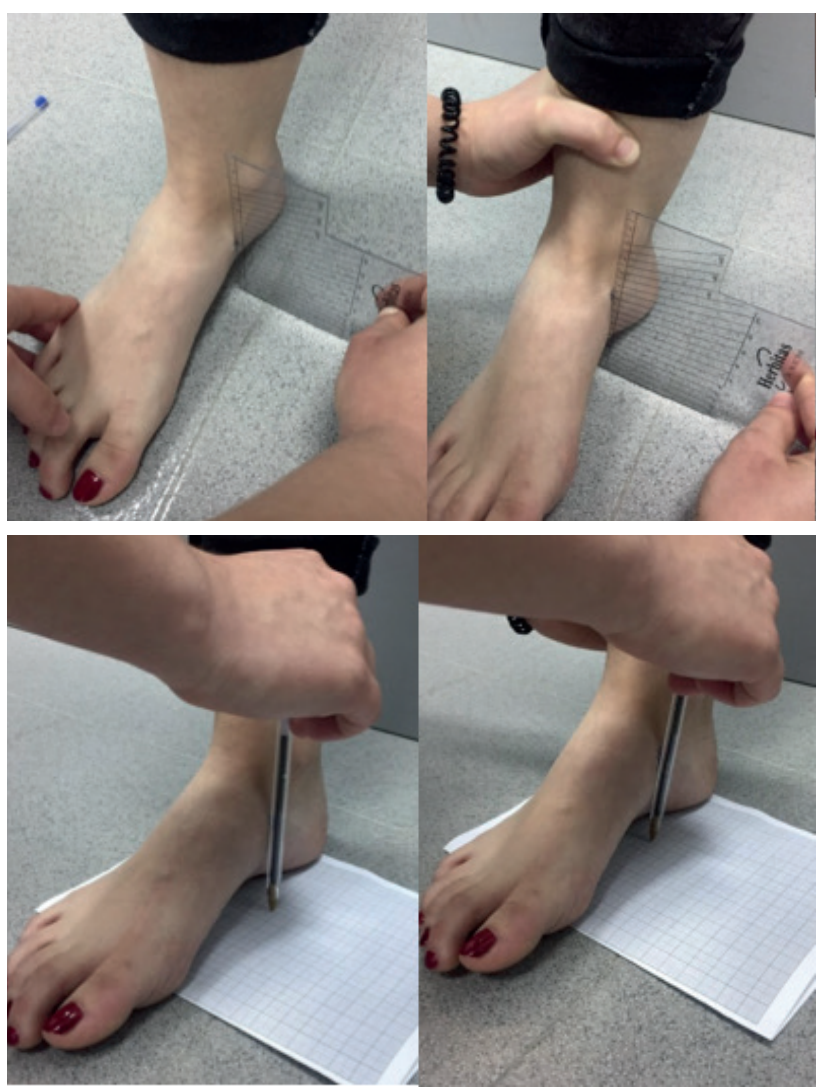

Figura 2. Test navicular drop (arriba) y navicular drift (abajo).

\section{Análisis de los datos}

Para el análisis de los datos se utilizó el programa estadístico SPSS versión 24.0 para Windows 10. Se realizó un análisis estadístico mediante la prueba t de student con un intervalo de confianza del $95 \%$, para comparar las medias de ambos sexos. Por otro lado, se realizaron correlaciones bivariadas mediante el coeficiente de correlación Pearson entre las variables test de Lunge, FPI, NDrop y NDrift, con el objetivo de observar si existían relaciones significativas entre ellas. Se estimaron valores estadísticamente significativos cuando el valor $p$ de la prueba de correlación de Pearson fue menor o igual a 0,05.

\section{RESULTADOS}

Se incluyeron un total de 57 sujetos en el estudio. En la Tabla I se muestran los datos antropométricos de los sujetos del estudio. La Tabla II muestra la frecuencia y porcentajes de las variables cualitativas del estudio, incluido los resultados del IPP. Se realizó un análisis descriptivo de la muestra. De los 57 sujetos, el $38.59 \%$ eran hombres y el $61.40 \%$ mujeres. En el estudio se analizaron un total de 114 pies. El $44.73 \%$ de los participantes presentaban pies normales, el $28.94 \%$ pies pronados y el $26.31 \%$ pies supinados.

Se realizó un análisis estadístico de las variables. Primero se analizó si había diferencias significativas entre hombres y mujeres respecto al IPP, test de Lunge, NDrop y NDrift del pie derecho (PD) y pie izquierdo (PI). Según los resultados obtenidos, no se puede asumir que existan diferencias significativas entre hombres y mujeres respecto al IPP, test de Lunge, Ndrop y NDrift (Tabla II). Se investigó si existía relación entre el IPP, el test de Lunge y el NDrop y NDrift mediante el coeficiente de correlación de Pearson. Tras los resultados obtenidos (Tabla III), se observó que existía una

\section{Tabla I. Medias y desviación típica de los valores} antropométricos de los sujetos de estudio

\begin{tabular}{llll} 
Edad (años) & Peso $(\mathrm{kg})$ & Talla $(\mathrm{m})$ & Total sujetos $(\mathrm{n})$ \\
\hline $22.45 \pm 4.73$ & $62.63 \pm 4.73$ & $1.60 \pm 1.26$ & 57 \\
\hline
\end{tabular}

Tabla II. Media y desviación típica de las variables del estudio

\begin{tabular}{lllll} 
Pie & IPP & Test de Lunge & NDrop & NDrift \\
\hline Hombres PD & $2.32 \pm 1.359$ & $26.68 \pm 9.03$ & $0.43 \pm 0.19$ & $0.40 \pm 0.18$ \\
\hline Mujeres PD & $1.91 \pm 1.136$ & $29.02 \pm 10.58$ & $0.46 \pm 0.25$ & $0.32 \pm 0.14$ \\
\hline Hombres PI & $2.32 \pm 1.359$ & $27.54 \pm 9.13$ & $0.52 \pm 0.054$ & $0.40 \pm 0.17$ \\
\hline Mujeres PI & $1.91 \pm 1.136$ & $27.54 \pm 7.022$ & $0.54 \pm 0.31$ & $0.35 \pm 0.16$ \\
\hline
\end{tabular}

IPP: índice de la postura del pie. NDrop: navicular drop. NDrift: navicular drift. PD: pie derecho. PI: pie izquierdo. 
Tabla III. Coeficiente de correlación de Pearson

\begin{tabular}{llll} 
IPP & Test de Lunge & NDrop & NDrift \\
\hline PD & $0.094>0.05$ & $0.003<0,01$ & $0.019<0.05$ \\
\hline PI & $0.062>0.05$ & $0.016<0.05$ & $0.007<0.01$ \\
\hline Test de Lunge & NDrop & NDrift & \\
\hline PD & $0.015<0.05$ & $0.031<0.05$ & \\
\hline PI & $0.040<0.05$ & $0.045<0.05$ & \\
\hline
\end{tabular}

IPP: índice de la postura del pie. NDrop: navicular drop. NDrift: navicular drift. PD: pie derecho. PI: pie izquierdo.

correlación baja y positiva entre el IPP y el NDrop del PD, con un valor $p=0.003(r=0.384 ; p<0.01)$, y del $\mathrm{PI}$, con un valor $p=0.016(r=0.310 ; p<0.05)$. Por otro lado, existe una correlación baja y positiva entre el FPI y el NDrift del PD, con un valor $p=0.019(r=0.310 ; p<0.05)$, y del PI, con un valor $p=0,007(r=0.356 ; p<0.01)$. Sin embargo, no existe relación entre las variables IPP y test de Lunge, con un valor $p=0.094$ para el PD y un valor $p=0.062$ para el izquierdo ( $p>0.05$ para ambos pies).

Se observó que existe una correlación baja y negativa entre el test de Lungey el Ndrop del PD, con un $p=0.015(r=-0.321$; $p<0.05)$, y del PI, con un valor $p=0.040(r=-0.273 ; p<0.05)$. Por otro lado, se encontró una correlación baja y negativa entre el test de Lunge y el NDrift del PD, con un valor $p=0.031$ $(r=-0.253 ; p<0.05)$, y del PI, con un $p=0.045(r=-0.287$; $p<0.05)$.

\section{DISCUSIÓN}

El objetivo del presente estudio fue analizar si existe relación entre la FD de tobillo, la flexibilidad de la columna medial y la postura del pie. El tobillo y la columna medial son de suma importancia para el correcto funcionamiento de la marcha humana. Es necesario que exista un correcto rango de movilidad articular para efectuar una adecuada dinámica del cuerpo humano. En la exploración biomecánica normalmente se valora la FD de tobillo en descarga, pero estudios recientes describen el test de Lunge como una prueba de elevada fiabilidad intraevaluador e interevaluador ${ }^{3,4,6,9}$. Otra prueba muy utilizada para clasificar el tipo de pie que presenta el paciente en carga es el IPP.

La valoración de la FD de tobillo en carga ha sido uno de los aspectos más relevantes de nuestro estudio, junto con el análisis de la flexibilidad de la columna medial. Esto se debe a que la FD de tobillo tiene gran importancia en las actividades de carga, y su estudio es de gran importancia en la literatura. Algunos autores, como Cejudo y cols. ${ }^{5}$, Alfaro Santafé y cols. ${ }^{3}$, Hall y cols. ${ }^{6}$ y Langarika-Rocafort y cols. ${ }^{9}$, reflejan la importancia de la valoración del tobillo en carga debido a que es la mejor forma de observar cómo se comporta esta articulación en dinámica. En estos estudios se nombra el test de Lunge como método o prueba más fiable para la valoración de la FD de la ATPA en carga.

Otro aspecto importante para la dinámica y las actividades en carga del pie es observar cómo influye la postura del pie en la flexibilidad de la columna medial y si existe una correlación entre ambos parámetros, ya que diferentes autores indican que la postura del pie puede afectar a la flexibilidad de la columna interna predisponiendo al sujeto a padecer determinadas patologías, como fascitis plantar, disfunción tibial posterior y miositis del abductor del hallux, entre otras $3,5,7,24$. En este estudio se encontró una correlación entre el IPP y el NDrop, la cual refleja la existencia de una relación lineal baja y directa entre ambos parámetros. Esto implica que cuanto mayor sea el valor del IPP, más descenso existe del navicular; por lo tanto, cuanto más pronado esté el pie, mayor será el descenso del navicular. Este estudio está de acuerdo con las investigaciones de Cowley y Marsden ${ }^{18}$ y Kothari y cols. ${ }^{19}$ : a pesar de encontrar una correlación entre ambos parámetros, esta no es estadísticamente significativa, y estos autores indican que la baja relación existente se puede deber a que durante la valoración de los parámetros del IPP se tienen en cuenta las partes blandas y óseas del pie, mientras que en el caso del NDrop solo se analiza el navicular, por lo que al tener mayor número de parámetros en el IPP, en su conjunto estos no tienen por qué influir significativamente sobre el NDrop. Esta investigación también está de acuerdo con el estudio de Langley y cols. ${ }^{20}$, pero en este caso los autores indican que el NDrop por sí solo no se debería utilizar para la clasificación del pie, si no que debería relacionarse con el IPP. Por otro lado, el estudio de Peláez Menacho y cols. ${ }^{21}$ contradice lo hallado en esta investigación, ya que no se encontró relación entre el IPP y el NDrop, y explican que puede influir igualmente lo citado antes para el estudio de Cowley y Marsden ${ }^{18}$; es decir, puede que no se encuentre una relación entre el IPP y el NDrop debido a que las variables que se analizan son distintas. Por otro lado, Kothari y cols. ${ }^{19}$ no encontraron una relación significativa entre NDrop e IPP.

Respecto a la relación entre el IPP y NDrift, este estudio refleja que existe una relación baja y directa entre ambos. Esto implica que cuanto mayor sea el IPP del pie, mayor será el valor del NDrift, lo que se traduce como a mayor pronación del pie, mayor es el desplazamiento del navicular entre la posición relajada del calcáneo en apoyo y la posición neutra. Se ha encontrado una similitud con la investigación de Kothari y cols. ${ }^{19}$. Existen escasas publicaciones recientes que evalúen y utilicen el NDrift, por lo que es difícil comparar los resultados con otras investigaciones de carácter relevante.

No se ha encontrado diferencias estadísticamente significativas en el IPP entre hombres y mujeres. Otros autores como Cowley y Marsden ${ }^{18}$ y Jiménez-Cebrián y cols. ${ }^{22}$ también afirman que el sexo no influye en la postura del pie.

Los resultados obtenidos para el test de Lunge con respecto al NDrop y NDrift reflejan una relación estadísticamente signi- 
ficativa. Nuestros resultados están en la línea de los resultados publicados por Chimenti y cols. ${ }^{24}$. Para ellos, la FD del tobillo y el NDrop se encuentran relacionados. Los sujetos con bajo rango de FD de tobillo presentan una disminución en la altura del ALI y un incremento del descenso del navicular. Un posible mecanismo que explique todo lo comentado anteriormente es que cuanto menor es la FD de la ATPA, mayor es la pronación de la articulación subastragalina, y esto se puede traducir como un mayor aplanamiento del ALI que puede provocar un descenso del navicular. Parece que una disminución de la FD de tobillo puede influir en la rigidez de la columna medial, siendo esta menos rígida.

En cuanto a la relación del test de Lunge con el NDrift, el presente estudio ha encontrado una relación estadísticamente significativa, pudiendo afirmar que existe una relación lineal e inversa entre ambos parámetros. No se han encontrado estudios recientes que lleven a cabo el análisis de ambas variables y las relacionen entre sí, por lo que no se puede realizar una comparativa de este estudio con otros de relevancia científica.

Los hallazgos del presente estudio describen parámetros biomecánicos del miembro inferior que pueden ser útiles para la realización de exploraciones biomecánicas en la clínica. En el caso del test de Lunge, se puede utilizar como test complementario a la valoración del tobillo en descarga, ya que puede haber un cambio respecto a esta articulación dependiendo de si el sujeto se encuentra en descarga o en bipedestación estática. Además, la valoración de las articulaciones tanto en carga como en descarga permite conocer los cambios fisiológicos que sufre el pie. Los resultados de este estudio parecen explicar los hallazgos clínicos que encontramos en patologías que afectan a la columna medial, como la fasciopatía plantar o la disfunción de tibial posterior, entre otros.

La muestra del siguiente estudio pertenece a la población adulta y la gran mayoría son jóvenes sin patologías. Por esta razón no se pueden generalizar los resultados para la población pediátrica y geriátrica, ni para individuos que padezcan alguna patología del miembro inferior (esguinces, hallux valgus, fascitis plantar, etc.). Pensamos que sería interesante realizar este tipo de estudio en gente de mayor edad, para observar si el envejecimiento es un factor que influye en la FD de la ATPA y en la flexibilidad de la columna interna. Se debería realizar también con gente con patologías del pie y el tobillo, para ver si existe correlación con la aparición de patologías como la fascitis plantar y el hallux rigidus. Otra limitación del estudio es que el tamaño de la muestra es reducido, por lo que puede ocurrir que no exista un predominio de un tipo de pie tanto en hombres como en mujeres. Para futuros trabajos de investigación se puede seguir analizando el comportamiento biomecánico de la articulación de tobillo y la columna medial en un tamaño de muestra mayor al del presente estudio, y también realizar un análisis en sujetos que presenten patologías del pie así como en población pediátrica y geriátrica.
En conclusión, el presente estudio ha encontrado correlación entre el rango de flexión dorsal de tobillo y la estabilidad de la columna medial medida clínicamente, y entre la posición del pie y la estabilidad de la columna medial en sujetos sanos. Se ha encontrado que a mayor flexión dorsal de tobillo, parece existir menor flexibilidad de la columna medial. Se debería seguir investigando el comportamiento biomecánico de la articulación de tobillo y de la columna medial por su relación con la marcha humana, así como la efectividad de las diferentes pruebas descritas para analizar estos parámetros.

\section{CONFLICTO DE INTERESES}

Los autores no presentan conflictos de interés relevantes con el presente artículo.

\section{FINANCIACIÓN}

Ninguna.

\section{BIBLIOGRAFÍA}

1. Sanchis Sales E. Influencia de la postura del pie sobre la rigidez de la columna medial durante la marcha [tesis doctoral]. Castellón: Universitat Jaume I; 2017.

2. Sánchez Monzó C, Fuertes Lanzuela M, Ballester Alfaro JJ. Inestabilidad crónica de tobillo: actualización. Rev Soc And Trauma Ortoped. 2015;32(2):19-29.

3. Alfaro Santafé JJ, Gómez Bernal A, Lanuza Cerzócimo C, Sempere Bonet C, Barniol Mercade A, Alfaro Santafé JV. Resultados del test de Lunge en pacientes con hallux limitus funcional: estudio transversal de casos y controles. Rev Esp Podol. 2017. 28(2):87-92. DOI: 10.1016/j. repod.2017.10.001.

4. Williams CM, Caserta AJ, Haines TP. The TiltMeter app is a novel and accurate measurement tool for the weight bearing lunge test. J Sci Med Sport. 2013;16(5):392-5. DOI: 10.1016/j.jsams.2013.02.001.

5. Cejudo A, Sainz de Baranda P, Ayala F, Santonja F. A simplified version of the weight-bearing ankle lunge test: Description and test-retest reliability. MT. 2014;19(4):355-9. DOI: 10.1016/j.math.2014.03.008.

6. Hall EA, Docherty CL. Validity of clinical outcome measures to evaluate ankle range of motion during the weight-bearing lunge test. J Sci Med Sport. 2017;20(7):618-21. DOI: 10.1016/j.jsams.2016.11.001.

7. Jung D-Y, Koh E-K, Kwon O-Y, Yi C-H, Oh J-S, Weon J-H. Effect of medial arch support on displacement of the myotendinous junction of the gastrocnemius during standing wall stretching. J Orthop Sport Phys Ther. 2009;39(12):867-74. DOI: 10.2519/jospt.2009.3158.

8. Barouk LS, Barouk P. Gastrocnemio cortos. Revista del pie y tobillo. 2012;2:7-13.

9. Langarika-Rocafort A, Emparanza JI, Aramendi JF, Castellano J, Calleja-González J. Intra-rater reliability and agreement of various methods of measurement to assess dorsiflexion in the Weight Bearing Dorsiflexion Lunge Test (WBLT) among female athletes. PT in Sport. 2017;23 (Supplement C):37-44. DOI: 10.1016/j.ptsp.2016.06.010.

10. Bennell K, Talbot R, Wajswelner H, Techovanich W, Kelly D, Hall A. Intra-rater and inter-rater reliability of a weight-bearing lunge measure of ankle dorsiflexion. Aust J Physiother. 1998;44(3):175-80. DOI: 10.1016/S0004-9514(14)60377-9.

11. Kirby KA. Biomecánica del pie y la extremidad inferior IV. Arizona: Precision Intricast; 2016. p. 27-38.

12. Bjelopetrovich A, Barrios JA. Effects of incremental ambulatory-range loading on arch height index parameters. J Biomech. 2016;49(14):35558. DOI: 10.1016/j.jbiomech.2016.08.017. 
13. Allen MK, Glasoe WM. Metrecom measurement of navicular drop in subjects with anterior cruciate ligament injury. J Athl Train. 2000;35(4):403-6.

14. Vauhnik R, Turk Z, Pilih IA, Mičetić-Turk D. Intra-rater reliability of using the navicular drop test for measuring foot pronation. Hrvatski Športskomedicinski Vjesnik. 2006;21(1):8-11.

15. Cavanagh PR, Rodgers M, Virginia W. Technical note the Arch Index: A useful measure. J Biomech. 1986;20(5):547-51. DOI: 10.1016/00219290(87)90255-7.

16. Menz HB. Alternative techniques for the clinical assessment of foot pronation. J Am Podiatr Med Assoc. 1998;88 (3):119-29. DOI: 10.7547/87507315-88-3-119.

17. Nielsen RG, Rathleff MS, Simonsen $\mathrm{OH}$, Langberg $\mathrm{H}$. Determination of normal values for navicular drop during walking: A new model correcting for foot length and gender. J Foot Ankle Res. 2009;2(1):1-7. DOI: 10.1186/1757-1146-2-12.

18. Cowley E, Marsden J. The effects of prolonged running on foot posture: A repeated measures study of half marathon runners using the foot posture index and navicular height. J Foot Ankle Res. 2013;6(1):1-6. DOI: 10.1186/1757-1146-6-20.

19. Kothari A, Dixon PC, Stebbins J, Zavatsky AB, Theologis T. Motion analysis to track navicular displacements in the pediatric foot: relationship with foot posture, body mass index, and flexibility. Foot Ankle Int. 2014;35(9):929-37. DOI: 10.1177/1071100714537629.

20. Langley B, Cramp M, Morrison SC. Clinical measures of static foot posture do not agree. J Foot Ankle Res. 2016;9(1):1-6. DOI: 10.1186/ s13047-016-0180-3

21. Peláez Menacho A, Parra Cortés L, Munuera Martínez PV. Cambios en la postura del pie tras la actividad deportiva en ciclistas de montaña masculinos: estudio piloto. Rev Esp Podol. 2016;27(1):10-7. DOI: 10.1016/j.repod.2016.05.005.

22. Jiménez-Cebrián AM, Morente-Bernal MF, Román-Bravo PD, Saucedo-Badía JF, Alonso-Ríos JA, Montiel-Luque A. Influence of age, sex, and anthropometric determinants on the foot posture index in a pediatric population. J Am Podiatr Med Assoc. 2017;107(2):124-9. DOI: 10.7547/14-097.

23. Sanchis Sales E, Sancho Bru JL, Rosa Sales A, Pascual Huerta J. Effect of static foot posture on the dynamic stiffness of foot joints during walking. Gait Posture. 2018;62:241-6. DOI: 10.1016/j.gaitpost.2018. 03.028 .

24. Chimenti RL, Forenza A, Previte E, Tome J, Nawoczenski DA. Forefoot and rearfoot contributions to the lunge position in individuals with and without insertional Achilles tendinopathy. Clin Biomech. 2016;36:40-5. DOI: 10.1016/j.clinbiomech.2016.05.007 